\title{
Alternaria sensitisation at age 6 years is associated with subsequent airway hyper-responsiveness in non- asthmatics
}

\author{
Anunya Hiranrattana, ${ }^{1}$ Debra A Stern, ${ }^{2}$ Stefano Guerra, ${ }^{2}$ Marilyn Halonen, ${ }^{2}$ \\ Anne L Wright, ${ }^{1,2}$ Michael Daines, ${ }^{1,2}$ Fernando D Martinez, ${ }^{2}$ Wayne J Morgan ${ }^{1,2}$
}

${ }^{1}$ Pediatric Pulmonary Allergy Division, Department of Pediatrics, University of Arizona, Tucson, Arizona, USA

${ }^{2}$ Asthma and Airway Disease Research Center, University of Arizona, Tucson, Arizona, USA

\section{Correspondence to}

Professor Wayne J Morgan, Asthma and Airway Disease Research Center, University of Arizona, Tucson, AZ 85724, USA;

wjmorgan@email.arizona.edu

Received 28 March 2017 Revised 19 February 2018 Accepted 26 February 2018 Published Online First 21 March 2018

\section{Check for updates}

To cite: Hiranrattana $A$, Stern DA, Guerra $S$, et al. Thorax 2018;73:1170-1173.

\section{ABSTRACT}

In the non-selected birth cohort Tucson Children's Respiratory Study, early sensitisation to Alternaria was associated with increased airway hyper-responsiveness (AHR) into adult life among non-asthmatics. The increase in AHR was of a similar magnitude to that seen for Alternaria sensitised asthmatics and was primarily evident among those who were overweight or obese. In contrast, there was no significant association between early sensitisation to aeroallergens other than Alternaria and AHR among non-asthmatics. Why this group of Alternaria sensitised individuals without asthma demonstrated increased AHR of a magnitude similar to asthmatics is unknown and requires further investigation.

\section{INTRODUCTION}

Early sensitisation to the fungus Alternaria and airway hyper-responsiveness (AHR) to cold air challenge at 6 years of age are associated with chronic asthma in early adult life. ${ }^{1}$ Among asthmatics, Alternaria sensitisation, in particular, is associated with risk for asthma persistence, severity and potentially fatal asthma exacerbations. ${ }^{2}$ In one of the few population-based cohort studies to have compared clinical characteristics of children sensitised to Alternaria with those sensitised to other allergens, Downs and colleagues showed that children sensitised to Alternaria were more likely to have AHR than were children sensitised to other allergens (OR 1.26) and the association was strengthened with increased Alternaria exposure. ${ }^{3}$ In our region, Alternaria is ubiquitous and a known asthma associated aeroallergen similar to house dust mites in other locations with higher humidity or cockroaches in the inner city environment. ${ }^{4}$ The relationship of obesity to AHR in asthmatics and non-asthmatics is contradictory. In asthmatics, body mass index (BMI) was negatively associated with AHR or showed no association..$^{5-8}$ In contrast, studies among non-asthmatics show a positive association of BMI to AHR. ${ }^{910}$ To our knowledge, there is limited information about the relation of Alternaria sensitisation to airway responsiveness among non-asthmatics and whether there are factors that modify this relation, such as obesity.

\section{METHODS}

Data from the Tucson Children's Respiratory Study, a non-selected birth cohort of healthy infants enrolled between 1980 and 1984, were used to investigate the relationship between
Alternaria sensitisation and AHR based on response to methacholine challenge in individuals with and without asthma. Participants completed skin prick tests (SPT) at age 6 to Alternaria alternata $(1: 100 \mathrm{w} / \mathrm{v})$, Bermuda grass (Cynodon dactylon, 1:20 w/v), careless weed (Amaranthus palmeri, $1: 20 \mathrm{w} / \mathrm{v})$, house dust mix $(1: 10 \mathrm{w} / \mathrm{v})$ and olive (Olea europaea), mesquite (Prosopis glandulosa) and mulberry trees (Morus alba) (1:20w/v) (Hollister-Stier Laboratories, Everett, Washington, USA). SPT responses at age 6 were grouped into three categories based on AAAAI practice guidelines ( $\geq 3 \mathrm{~mm}$ average weal size minus negative control): Alternaria-positive (regardless of other sensitisation, 'Altpos'), non-Alternaria-positive (positive to allergens other than Alternaria, 'non-Altpos') and SPT negative ('SPTneg'). Skin prick testing was repeated at ages 11, 16, 22 and 26. Of the allergens tested at age 6, Alternaria, Bermuda, careless weed, olive, mesquite and mulberry were tested again at these ages.

Spirometry and methacholine challenge were performed at ages 11, 16, 22 and 26. A controlled inhalation protocol using a dosimetric method modified after Chai et al. ${ }^{11}$ was used for the methacholine challenges. This protocol doubles the cumulative methacholine dose from 0.004 to $2.048 \mathrm{mg}$ with the endpoint defined as a 20\% drop in FEV1 or completion of the final dose. The dose response slope was calculated as described by Chinn et $a l^{12}$ and Marcon et al. ${ }^{13}$ Briefly, the per cent decrease in FEV1 from baseline was regressed on log10 dose (mg) using all data except postsaline FEV1. Baseline FEV1 was included in the calculation of the slope and assigned a per cent decrease of ' 0 ' and an arbitrary dose of $0.002 \mathrm{mg}$, the next lowest stepwise dose before $0.004 \mathrm{mg}$. The resulting 'log-slope' then underwent a shifted reciprocal transformation as described by Chinn $(100 /(\log \text {-slope }+10))^{12}$ and is hereafter referred to as the 'log-slope'. Lower levels of the transformed log-slope correspond to increased airway responsiveness. We also calculated the provocative dose 20 (PD20) at each age using the formula described by Dell et al. ${ }^{14}$ The median (min, max) methacholine log-slope for those with a PD20 between $0.512 \mathrm{mg}$ and $1.024 \mathrm{mg}$ was 5.52 $(4.8,6.1), 5.45(4.9,5.9), 5.58(4.2,6.3)$ and 5.43 $(4.5,6.6)$ at ages $11,16,22$ and 26 , respectively. Total serum IgE was measured at these same ages and values were log base 10 transformed.

A report of a physician diagnosis of asthma with active symptoms during the past year was 
ascertained from questionnaires completed at ages $6,8,11,13$, $16,18,22,24,26$ and 29 years. Asthma questions were similar at each age, for example, at age 22 participants were asked: 'Have you ever had asthma (reactive airways disease)?'. If an affirmative response was provided, additional questions included 'Did a doctor ever say you had asthma?' and 'During the past year, how many asthma attacks or episodes did you have?'. A participant was classified as 'asthmatic' if they ever reported an asthma diagnosis with active symptoms during the past year at any age and were classified as 'non-asthmatic' if they completed one or more questionnaires and did not report asthma. Active wheeze was defined as wheeze within the previous 12 months obtained from questionnaires using similar questions at each age, for example at age 22 participants were asked: 'Has your chest ever sounded wheezy or whistling?' and if an affirmative response was provided, they were additionally asked 'During the past year, how often have you had wheezing or whistling?'. Overweight/obese was defined as a BMI at or above the 85 th percentile in children or at or above $25 \mathrm{~kg} / \mathrm{m}^{2}$ in adults.

Analysis was limited to participants with skin test data at age 6 and at least one subsequent methacholine challenge test. Relations between log-slope AHR and the asthma/SPT groups were assessed using one-way analysis of variance with Scheffe multiple comparison test, multiple regression and longitudinal random effects models adjusted for sex and concurrent FEV1, height and age. In the random effects models, survey year was included as a categorical variable.

\section{RESULTS}

There were 558 participants included in this analysis of which $33 \%(n=182)$ had physician diagnosed asthma that was active at one or more times between 6 and 29 years of age. Among non-asthmatics $(\mathrm{n}=376), 5 \%$ were Altpos $(\mathrm{n}=19), 15 \%$ were non-Altpos $(\mathrm{n}=58)$ and the remainder were SPTneg $(80 \%$, $\mathrm{n}=299)$ at age 6 years. Sensitisation at age 6 was more common among asthmatics, with 15\% Altpos $(\mathrm{n}=28), 24 \%(\mathrm{n}=43)$ non-Altpos and the remainder SPTneg $(61 \%, \mathrm{n}=111)$.

Both asthmatic and non-asthmatic Altpos participants were more likely to be polysensitised at age 6 compared with non-Altpos participants (table 1). Altpos and non-Altpos participants had higher total IgE at age 6 compared with SPTneg subjects. The majority of the Altpos participants at age 6 retained sensitisation to Alternaria when tested at subsequent ages $(65 \%$ at age $11,90 \%$ age $16,88 \%$ age $22 \%$ and $74 \%$ at age 26 years). Five participants were skin test positive to house dust mix at age 6: one in the Altpos non-asthmatic group, one in the Altpos asthmatic group and three in the non-Altpos asthmatic group.
Though intriguing that $4 / 5$ of the participants positive for house dust mix were in the asthma group, the analytical possibilities are limited by the small number of sensitised individuals in Tucson.

The methacholine response log-slope was used to assess AHR, lower values indicating increased AHR. When the methacholine challenge test results were expressed as a PD20, 76\%, 54\%, 56\% and $61 \%$ of the participants responded to methacholine with at least a 20\% drop in FEV1 from baseline at ages 11, 16, 22 and 26, respectively. The correlation between the log-slope and PD20 was strong at all ages $(\mathrm{rho}=0.90,0.85,0.88,0.86$ at ages 11, 16, 22 and 26, respectively); however, using the log-slope provides responsiveness estimates for all participants and does not censor those with a $<20 \%$ fall.

Altpos non-asthmatics had AHR of a similar magnitude to that seen for Altpos asthmatics (figure 1A,B). In an adjusted longitudinal model, AHR was increased in Altpos non-asthmatics $(\beta=-1.4,95 \% \mathrm{CI}-2.0$ to $-0.80, \mathrm{p}<0.001)$ and Altpos asthmatics $(\beta=-1.6,95 \% \mathrm{CI}-2.1$ to $-1.0, \mathrm{p}<0.001)$ compared with SPTneg non-asthmatics. While non-Altpos asthmatics had increased AHR compared with the SPTneg non-asthmatics $(\beta=-1.1,95 \% \mathrm{CI}:-1.5$ to $-0.7, \mathrm{p}<0.001)$, the AHR of non-Altpos non-asthmatics was similar to SPTneg non-asthmatics ( $\beta=-0.2,95 \% \mathrm{CI}-0.6$ to $0.2, p=0.236$ ), suggesting that sensitisation to allergens other than Alternaria is not associated with increased airway responsiveness among non-asthmatics.

When considered in a model limited to Altpos and non-Altpos non-asthmatics, AHR was significantly increased for Altpos compared with non-Altpos participants $(\mathrm{p}=0.002)$. To address the association of polysensitisation with Altpos, this model was adjusted for polysensitisation at age 6 and these same groups compared. Again, AHR was significantly increased for Altpos compared with non-Altpos $(\mathrm{p}=0.010)$. Similar results were seen after adjustment for concurrent total $\operatorname{IgE}(\mathrm{p}=0.002)$. These results suggest that among sensitised non-asthmatics, the increased AHR for Altpos is not due to polysensitisation or higher total IgE.

Among non-asthmatics $(n=375)$, Altpos and non-Altpos participants were more likely to wheeze between ages 11 and 26 compared with SPTneg participants (Relative risk $(\mathrm{RR})=2.1$, $95 \% \mathrm{CI}: 1.4$ to $3.1, \mathrm{p}<0.001$ and $\mathrm{RR}=1.7,95 \% \mathrm{CI} 1.3$ to 2.3 , $\mathrm{p}<0.001$, respectively), suggesting increased symptomatology in both atopic groups despite the lack of a diagnosis of asthma. However, non-asthmatic participants with active wheeze during the year prior to methacholine testing (ages 11, 16, 22 and 26) did not have significantly increased AHR compared with those without active wheeze $(\beta=-0.2,95 \% \mathrm{CI}-0.5$ to 0.01 , $\mathrm{p}=0.063$ ).

Table 1 Number of positive skin prick tests and total serum lgE levels for the skin prick test groups at age 6 years stratified by physician diagnosed (MD) asthma 6-29 years

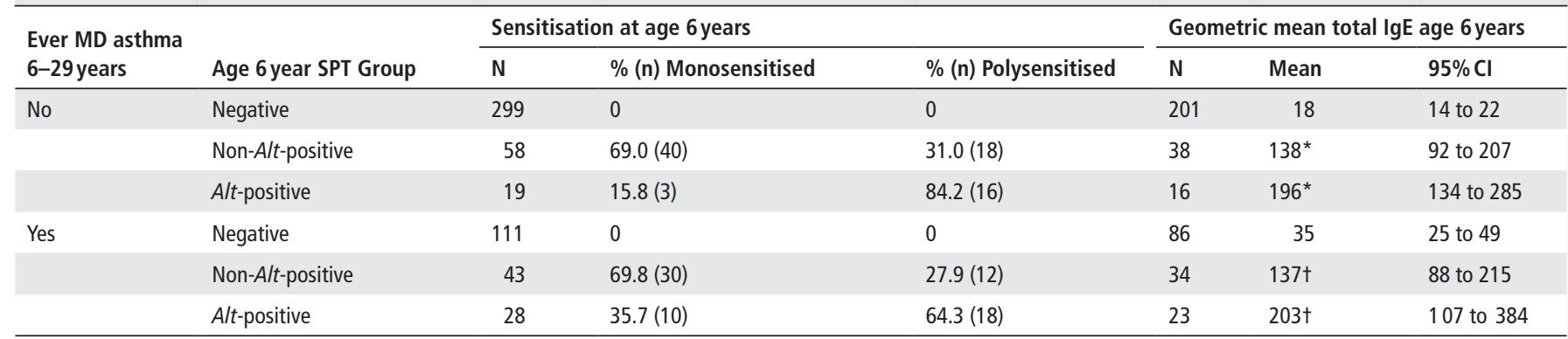

${ }^{*} \mathrm{P}<0.05$ compared with non-asthmatic SPTneg.

$+\mathrm{P}<0.05$ compared with asthmatic SPTneg. 

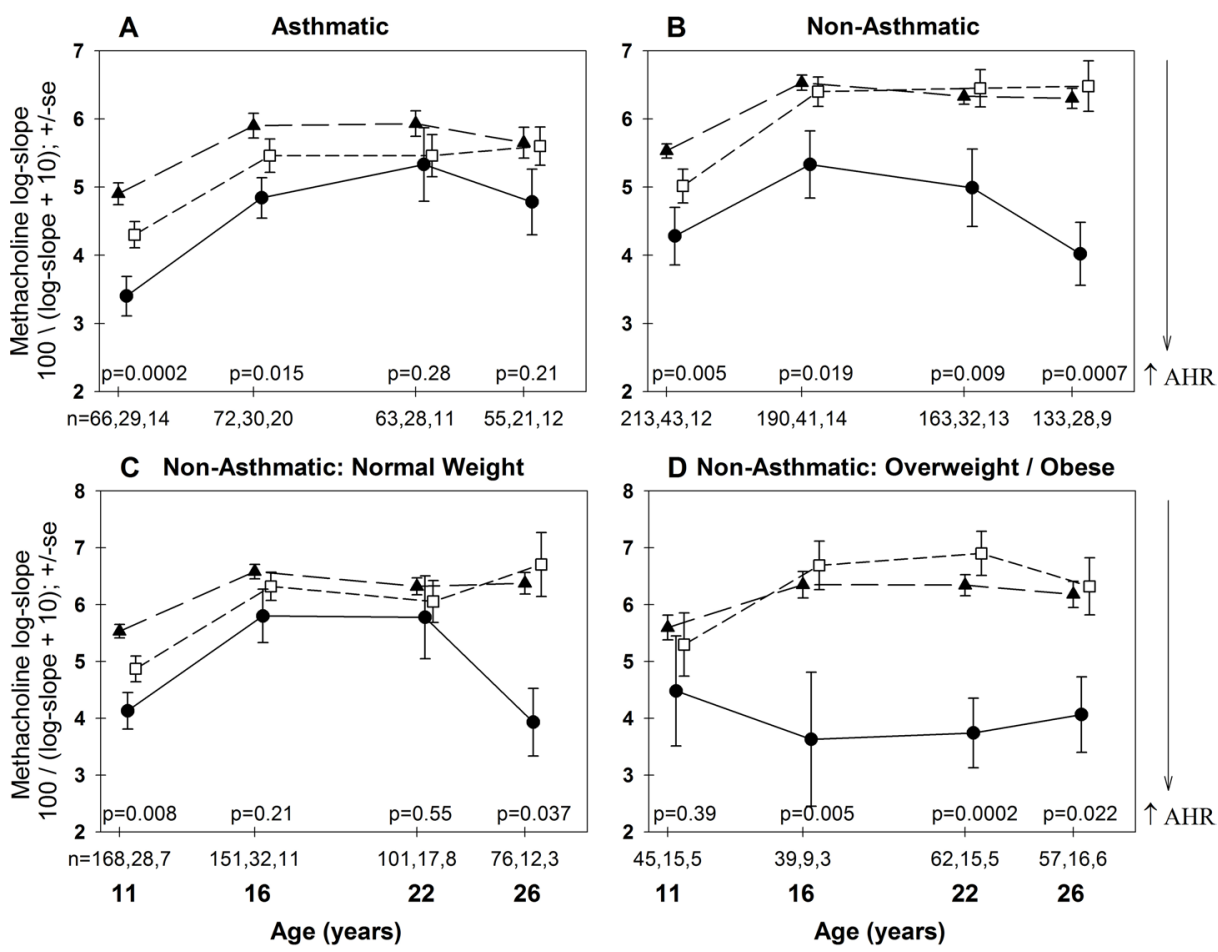

D Non-Asthmatic: Overweight / Obese
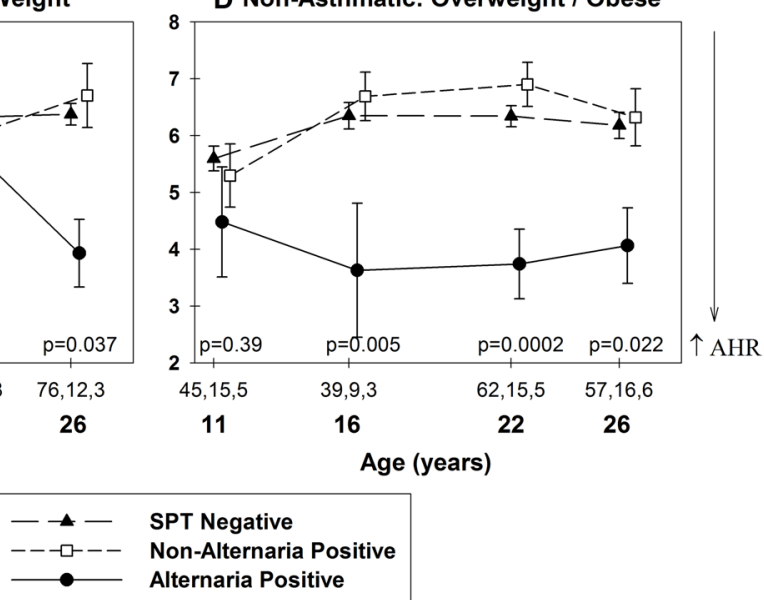

Figure 1 Methacholine log-slope at ages 11-26 years categorised by SPT groups at 6 years of age for asthmatic (A) and non-asthmatic participants (B). Among non-asthmatic participants, the relation between SPT groups at age 6 and subsequent airway hyper-responsiveness was further divided into normal weight (C) and overweight/obese (D) participants. Unadjusted ANOVA performed at each age and the age-specific $p$ values and number of participants reported for each group are listed as: SPT, non-Alternaria-positive, Alternaria-positive. AHR, airway hyper-responsiveness; ANOVA, analysis of variance; SPT, skin prick test.

In order to address the effects of possible under-reporting of asthma, we examined the relation of sensitisation to AHR among non-asthmatics limiting the analysis to those who did not report active wheeze during the year prior to methacholine testing (ages 11, 16, 22 and 26). In this subset, $n=339$, AHR was increased in Altpos non-asthmatics $(\beta=-1.3,95 \% \mathrm{CI}-2.0$ to- $-0.6, \mathrm{p}<0.001)$ compared with SPTneg non-asthmatics. AHR was similar for non-Altpos non-asthmatics $(\beta=-0.2,95 \% \mathrm{CI}-0.6$ to 0.2 , $\mathrm{p}=0.338$ ) compared with SPTneg non-asthmatics. Thus, results were similar for non-wheezing non-asthmatics as for the whole group of non-asthmatics.

On further longitudinal analysis among non-asthmatics, the increased AHR among Altpos participants compared with the SPTneg group was significant for normal weight $(\beta=-0.9$, $95 \% \mathrm{CI}-1.7$ to $-0.2, \mathrm{p}=0.012$ ) and stronger for overweight/ obese individuals $(\beta=-2.0,95 \% \mathrm{CI}-2.9$ to $-1.0, \mathrm{p}<0.001)$ in adjusted longitudinal models (figure 1C,D). The interaction of overweight/obese with Altpos was statistically significant among non-asthmatics, $\mathrm{p}=0.007$, suggesting an enhanced risk for AHR among overweight/obese individuals even with the caveat of small group sizes. This interaction was still significant after adjusting for BMI at age 6 years $(p=0.010)$ and adjusting for wheeze during the year prior to methacholine testing $(\mathrm{p}=0.007)$. Non-Altpos sensitisation at age 6 was unrelated to AHR in either weight group. With small numbers in any given subset and a maximum of only four data points per participant, these longitudinal analyses should be interpreted with caution.

\section{DISCUSSION}

Among non-asthmatic participants, Alternaria sensitisation at age 6 years was associated with increased airway responsiveness from ages 11 to 26 that was of a similar magnitude to that seen in participants who had asthma. Because of the strong association of Alternaria sensitisation with asthma, the number of Alternaria-sensitised individuals who did not develop asthma is small (19/47). Nevertheless, the association between early sensitisation and increased airway reactivity is consistent at 16, 22 and 26 years.

Several previous studies have identified non-asthmatic individuals who have methacholine AHR. ${ }^{15} 16$ Almost invariably, these studies relied on the subject's recall to determine the past history of asthma. Here, we based asthma diagnosis on prospectively obtained data as part of a newborn cohort followed up to adult life. We confirmed that there are indeed adult subjects who did not have asthma during their lifetime but who still had AHR. We also showed, however, that sensitisation to Alternaria was strongly associated with AHR in these subjects. Laprise et al reported that adults with asymptomatic AHR were more likely to be atopic than asymptomatic subjects without AHR, had evidence of airway remodelling and were highly likely to develop asthma during the subsequent 2 years. ${ }^{16}$ Although it is not possible to know if these subjects might have forgotten that they had childhood asthma which was relapsing, it is tempting to speculate that atopy and continued exposure to certain perennial allergens may beget chronic airway inflammation. In the Tucson environment, Alternaria is an ideal candidate for such a 
scenario, given its ubiquity and high allergenicity. What remains unexplained is which factors protected our participants from developing asthma in spite of being sensitised in early life to the allergen most strongly associated with asthma in our locale and having persistent AHR.

Our data suggest that one factor that may influence protection from having asthma symptoms is obesity. We found a significant interaction between sensitisation to Alternaria and BMI as determinants of AHR in asymptomatic subjects. These results thus suggest that systemic inflammation ${ }^{17}$ and mechanical factors attributable to decreases in functional residual capacity and in tidal volume ${ }^{18}$ may enhance AHR in obese subjects. Late-onset asthma associated with obesity is most often non-atopic, ${ }^{19}$ but a recent retrospective study reported that obesity enhances the clinical expression of atopic asthma in adults. ${ }^{20}$ Continued follow-up of participants in our study may allow us to determine if obese subjects with AHR who are allergic to Alternaria are at increased risk for late-onset asthma.

Contributors Study concept and design: AH, WJM and FDM. Participant recruitment: ALW. Acquisition of data: FDM, WJM, ALW and MH. Analysis of data: DAS, AH, WJM, SG and FDM. Data interpretation and drafting of the manuscript: AH WJM, DAS, SG, MD, ALW, MH and FDM. All authors critically read, commented on and approved the final version of the manuscript.

Funding This work was supported by US Department of Health and Human Services, National Institutes of Health, National Heart Blood and Lung Institute, grant number 132523.

Competing interests None declared.

Patient consent Not required

Ethics approval This research was approved by the Institutional Review Board of the University of Arizona.

Provenance and peer review Not commissioned; externally peer reviewed.

(c) Article author(s) (or their employer(s) unless otherwise stated in the text of the article) 2018. All rights reserved. No commercial use is permitted unless otherwise expressly granted.

\section{REFERENCES}

1 Stern DA, Morgan WJ, Halonen M, et al. Wheezing and bronchial hyperresponsiveness in early childhood as predictors of newly diagnosed asthma in early adulthood: a longitudinal birth-cohort study. Lancet 2008;372:1058-64.
2 Denning DW, O'Driscoll BR, Hogaboam CM, et al. The link between fungi and severe asthma: a summary of the evidence. Eur Respir J 2006;27:615-26.

3 Downs SH, Mitakakis TZ, Marks GB, et al. Clinical importance of Alternaria exposure in children. Am J Respir Crit Care Med 2001;164:455-9.

4 Halonen M, Stern DA, Wright AL, et al. Alternaria as a major allergen for asthma in children raised in a desert environment. Am J Respir Crit Care Med 1997;155:1356-61.

5 Bustos $\mathrm{P}$, Amigo $\mathrm{H}$, Oyarzún $\mathrm{M}$, et al. Is there a causal relation between obesity and asthma? Evidence from Chile. Int J Obes 2005;29:804-9.

6 Del Rio-Navarro BE, Blandon-Vijil V, Escalante-Domínguez AJ, et al. Effect of obesity on bronchial hyperreactivity among Latino children. Pediatr Pulmonol 2013:48:1201-5.

7 Mansell AL, Walders N, Wamboldt MZ, et al. Effect of body mass index on response to methacholine bronchial provocation in healthy and asthmatic adolescents. Pediatr Pulmonol 2006;41:434-40.

8 Salome CM, Munoz PA, Berend N, et al. Effect of obesity on breathlessness and airway responsiveness to methacholine in non-asthmatic subjects. Int J Obes 2008;32:502-9.

9 Jang AS, Lee JH, Park SW, et al. Severe airway hyperresponsiveness in school-aged boys with a high body mass index. Korean J Intern Med 2006;21:10-14

10 Sood A, Verhulst SJ, Varma A, et al. Association of excess weight and degree of airway responsiveness in asthmatics and non-asthmatics. J Asthma 2006:43:447-52.

11 Chai H, Farr RS, Froehlich LA, et al. Standardization of bronchial inhalation challenge procedures. J Allergy Clin Immunol 1975:56:323-7.

12 Chinn S, Arossa WA, Jarvis DL, et al. Variation in nebulizer aerosol output and weight output from the Mefar dosimeter: implications for multicentre studies. Eur Respir J 1997; 10:452-6.

13 Marcon A, Cerveri I, Wjst M, et al. Can an airway challenge test predict respiratory diseases? A population-based international study. J Allergy Clin Immunol 2014; 133:104-10

14 Dell SD, Bola SS, Foty RG, et al. Provocative dose of methacholine causing a 20\% drop in FEV1 should be used to interpret methacholine challenge tests with modern nebulizers. Ann Am Thorac Soc 2015;12:357-63.

15 Blais CM, Davis BE, Cockcroft DW. Diversity of methacholine dose-response curves among asymptomatic non-asthmatics. Respir Med 2017;132:109-11.

16 Laprise C, Laviolette M, Boutet $M$, et al. Asymptomatic airway hyperresponsiveness: relationships with airway inflammation and remodelling. Eur Respir J 1999:14:63-73.

17 Zhang $X$, Zheng J, Zhang $L$, et al. Systemic inflammation mediates the detrimental effects of obesity on asthma control. Allergy Asthma Proc 2018:39:43-50.

18 Shore SA, Fredberg JJ, Obesity FJJ. Obesity, smooth muscle, and airway hyperresponsiveness. J Allergy Clin Immunol 2005;115:925-7.

19 Moore WC, Fitzpatrick AM, Li X, et al. Clinical heterogeneity in the severe asthma research program. Ann Am Thorac Soc 2013;10:S118-S124.

20 Fitzpatrick S, Joks R, Silverberg Jl. Obesity is associated with increased asthma severity and exacerbations, and increased serum immunoglobulin $\mathrm{E}$ in inner-city adults. Clin Exp Allergy 2012;42:747-59. 